\title{
LA RIBELLE DEL BALATON ${ }^{1}$
}

\author{
Adriana Assini ${ }^{2}$
}

Fiore d'inverno, ninfa sfuggente dal colorito lunare, Erzsébet Báthory, l'altera contessa padrona di cento castelli era la donna più potente del regno di Ungheria. Anche adesso che era rimasta vedova.

Bella, audace, insolente, annoverava personaggi importanti tra i suoi avi, come quel re di Polonia passato alla grande storia per aver combattuto e vinto Ivan IV di Russia, soprannominato il Terribile. Per questo, noncurante di leggi e consuetudini, aveva sempre mantenuto il suo cognome, anche dopo le nozze.

Educata nel rigore calvinista, Erzsi ondeggiava tra l'estrema severità e le improvvise stravaganze. Conosceva il latino, ne sapeva di medicina e praticava l'astrologia. Nonostante il lutto, vestiva spesso di rosso, facendosi notare per la spregiudicatezza dei pensieri e il suo andare sempre controvento.

Alle sue feste, una a ogni inizio di stagione, si ballava fino a tardi e a suonare chiamava spesso musicisti zigani, malgrado il fatto che quella razza fosse invisa agli invitati d'alto rango.

Ma c'era dell'altro: a Erzsi piaceva cavalcare sulle acque ghiacciate del Váh, bere liquori forti, occuparsi in prima persona di politica.

\footnotetext{
${ }^{1}$ Data di ricezione: 05/07/2013

Data di accettazione: 06/07/2013

${ }^{2}$ Adriana Assini ha pubblicato il suo primo romanzo Gilles Che amava Jeanne nel 1997 e da allora non ha smesso di scrivere: Nella foresta di Sonian, Lo scettro di seta, Il Fuoco e la creta, Il bacio del diavolo, Le rose di Cordova, Un sorso di arsenico, Il commerciante di zucchero, I racconti dell'ombra e Sogni di Vini. Alcuni romanzi e racconti sono stati tradotti in spagnolo. Ha ricevuto importanti premi, tra i quali: il Pavese, il Premio Accademia Terra Award Vesuvio e il Premio Parola di donna; adriana.assini@ancpl.it.
} 
"Onoro il drago che fiammeggia nel mio stemma" rispondeva a chi le rimproverava di immischiarsi in affari più adatti agli uomini, come la lotta per l'indipendenza del suo paese, ormai da tempo sotto il giogo della Corona Asburgica. "Se la libertà fosse un vizio, allora avreste ragione. Ma essendo una virtù, s'addice meglio alle donne...".

Quel giorno di un autunno precoce, il Conte Palatino si presentò nella sua residenza di Csejthe senza farsi preannunciare da un messo, cosa che mise subito in guardia la Contessa, capace di fiutarne in anticipo trappole e raggiri. Da quando, infatti, quell'uomo dai trascorsi brillanti s'era asservito a Vienna pur di assicurarsi prestigio, denaro e carriera, lei lo aveva privato del rispetto e della stima. Né le importava che Thurzó l'additasse come una spregiudicata solo perché poteva avvalersi di una milizia armata ed esercitava una certa influenza su molta parte della nobiltà locale.

Finora non l'aveva mai affrontata a viso aperto, ma adesso, gettata la maschera, s'era deciso a farle abbassare la cresta facendo valere tutta l'autorità che gli proveniva dalla sua alta carica.

"Oramai, i pettegolezzi su di voi non si contano. Se fossi al posto vostro, comincerei a temerli prima che si trasformino in terribili accuse...Purtroppo, il mondo è crudele e basta un'ora per infangare una reputazione costruita nei secoli" le disse rendendo forzato omaggio al suo casato.

Erzsébet non si scompose. Sapeva badare a se stessa e di certi consigli poteva farne a meno. Non scese a patti, non fece passi indietro.

“Alla reggia di Vienna si terrà un gran ballo per festeggiare l'avvento dell'inverno" la informò l'altro, invitandola a prendervi parte. "Stare lontana da qui per un po' potrebbe aiutarvi a ritrovare la lucidità perduta".

Abituata a dare battaglia, la Contessa respinse prontamente la proposta con una risata ironica. "Di questi tempi, caro György, succede spesso che i miei svaghi cedano il passo ai miei doveri" gli rispose con la solita sicumera, sottolineando che alle feste anteponeva sempre gli interessi del Paese. "Se vi dessi retta, dovrei chiamare i sarti per 
un nuovo abito da sfoggiare a corte. Io, invece, ho già convocato gli armaioli visto che mi sto preparando alla guerra" aggiunse minacciosa, non facendo mistero d'essere in attesa di una grossa fornitura di cotte di maglia, elmi e bombarde.

"Attenta! Ricordate cosa succede alle libellule quando volano verso il fuoco? Attratte dalla luce della fiamma, non percepiscono il pericolo e si bruciano. . ." cercò di intimidirla György Thurzó, indispettito da tanta boria.

"Fareste bene a non dimenticare mai chi sono" fece di rimando lei, con voce intinta nell'aceto. "Il nome che porto è già scritto nella Storia e non permetterò a nessuno, tanto meno a voi, di mettere in ginocchio la nostra nazione per miseri vantaggi personal".

Senza dargli modo di replicare, lo accusò di voler svendere la patria al re Mattia per puro tornaconto economico e sociale. Altrimenti, non si spiegava come avesse potuto stipulare un accordo che vedeva l'Ungheria ridotta al ruolo di vassalla dell'Impero Asburgico.

"Se foste saggia quanto siete fiera non parlereste con tanta imprudenza" provò ad azzittirla il Conte Palatino, che non si sentiva affatto un traditore, bensì un uomo avveduto, capace di valutare le sue forze e quelle altrui. "Con la vostra esperienza e la lungimiranza di cui andata nota, dovreste ormai saperlo che è meglio amare ciò che non siamo in grado di combattere...".

"L'avidità, purtroppo, vi rende cinico e cieco".

"Moderate le accuse se volete restare padrona in casa vostra. La mia pazienza non è infinita...".

Livido di collera, György interruppe l'alterco e senza un cenno di congedo s'avviò verso l'uscita, ma sul punto di varcare la soglia della grande sala degli affreschi, lanciò un ultimo anatema contro la sua acerrima nemica.

"So di Gábor. E anche questa è una faccenda che non mi piace affatto" disse chiamando in causa il nipote preferito della Báthory, giovane Principe della vicina 
Transilvania, col quale la nobildonna tentava di mettere a punto un'azione comune contro la Corona. "Non vorrei essere nei vostri panni il giorno in cui vi obbligherò a rispondere dei vostri complotti alle mie spalle..."

Noncurante delle minacce, sicura d'essere tuttora lei la più forte, Erzsi si limitò a indicargli l'uscita, poi andò a cercare Anna Darvulia, sua ancella e sua fedele consigliera, intenta a prepararle un nuovo intruglio con la biacca.

"Vi chiedo di sopportare ancora per un giorno, poi constaterete voi stessa i prodigi di questa polvere. Sbiancherà la vostra carnagione fino a farla sembrare più pallida della neve" promise quella non appena la vide comparire sull'uscio.

Ma lei, in quel momento, non aveva urgenza di fare invidia alle dee: "Malauguratamente, ho altro a cui pensare in questo momento! Scure nuvole minacciano i miei cieli..."

"Provo a indovinare: siete irritata perché Thurzó vi vorrebbe umile e inerme ai suoi piedi" fece Anna, che aveva visto il Conte arrivare in pompa magna e poi andarsene come una furia. "Ma perché amareggiarvi se non intendete obbedirgli?"

"Adesso ho la certezza che intercetta le mie lettere per conoscere i miei piani..." si sfogò la Contessa, che da troppi giorni non riceveva più la corrispondenza. Dettaglio inquietante, considerando che aspettava con ansia la risposta del nipote alla sua chiamata alle armi in difesa dell'autonomia della nazione.

"E se invece fosse stato Gabór a voltarvi le spalle? I giovani, si sa, sono ambiziosi quanto incostanti e lui ha dimostrato d'esserlo più degli altri..."

Che il principe della Transilvania non fosse uno stinco di stanco, Erzsi lo sapeva bene. Che mancasse agli appuntamenti coi suoi dignitari per trastullarsi con le donne, le era noto. Ciononostante, non voleva credere che fosse così sprovveduto da sottovalutare l'importanza della posta in gioco: fermare l'espansione di Vienna.

Fatto sta che in assenza di sue notizie, la Contessa non si decideva a prendere alcuna iniziativa, temendo di sbagliare qualche mossa e poi doversene pentire. Alla fine, 
Anna tentò di smuoverla da quell'immobilità che giudicava letale: con una piccola provocazione: "Forse è preferibile una resa onorevole a una sconfitta cocente..."

"Ritengo di dover abbassare la testa soltanto davanti al Padreterno. E confesso che neanche questo mi riesce sempre..." rispose Erzsébet con quell'antica fierezza che non celava nemmeno quando s'intratteneva in conversazioni mondane tra i suoi pari. Con le sue uscite forti, lasciava sempre tutti a bocca aperta e subito alle sue spalle piovevano $\mathrm{i}$ commenti, perché anche se alcuni l'ammiravano paragonandola a un valente condottiero, la maggioranza dei suoi interlocutori la deplorava, definendola empia e strafottente.

Adesso, stanca di rimestare in sterili pensieri, pregò l'ancella di interrogare per lei le stelle: "Questa notte stessa" disse. In base a ciò che gli astri le avrebbero predetto, avrebbe poi deciso le prossime mosse, senza il timore di mettere un piede in fallo.

Felice di servirla, qualche ora più tardi Anna Darvulia le tradusse i segni del cielo in presagi: "Il responso è chiaro: affinché le vostre impresse possano avere successo, dovrete sposare la prudenza dell'airone all'astuzia della volpe..."

Cosa che lei fece. Il giorno dopo convocò il comandante delle guardie e fu perentoria: "D'ora in avanti, che il ponte levatoio resti alzato anche durante il giorno. Le precauzioni non sono mai troppe in certi casi..."

"Il maltempo è dalla nostra parte, mia signora. Con questa neve, nessuno s'arrischierà a venire fin qui spingendo una bombarda..." la tranquillizzò il milite, escludendo a priori un attacco con le micidiali palle di ferro, che avrebbe sbriciolato le pur possenti mura del castello.

"Se il Conte dovesse attaccarmi, imparerà a sue spese che una donna sa dare battaglia quanto e più di un uomo" ripeteva a voce alta, convinta che avrebbe vinto la partita, forte anche degli insegnamenti militari ricevuti a suo tempo dal marito, quel Ferenc, detto il Cavaliere Nero, tuttora considerato grande guerriero ed eroe nazionale. 
Ma le stagioni si susseguirono senza che succedesse niente. Non un solo colpo sparato, né un agguato sventato. Di questo silenzio delle armi, lei non esultava né perdeva la calma, sapendo che l'attesa logora i nervi dei più deboli, non dei fini strateghi.

Il mese di dicembre portò la neve e fu freddo del solito. Pareva quasi che anche la natura si schierasse contro la Contessa, seppellendola assieme al borgo di Csejthe e alle valli circostanti sotto un manto glaciale.

Eppure, in quella situazione che avrebbe fatto sentire in trappola chiunque, lei non si scompose: imperturbabile, ripeteva ai suoi di stare tranquilli perché, a dispetto delle apparenze, era proprio nel cuore di quella gelida stagione che potevano ritenersi in una botte di ferro.

"Questo tempo da lupi ci mette al riparo da qualsiasi attacco. Nessuno sarà così stolto da inoltrarsi tra i ghiacci, a meno che non intenda crepare per gli stenti! E per quanto ci riguarda, abbiamo provviste in così tale abbondanza da poter banchettare fino a primavera senza privarci di niente..."

Tutto ciò non l'aveva comunque portata ad abbassare la guardia e le sue sentinelle, nonostante le temperature basse e un vento tagliente, avevano raddoppiato $\mathrm{i}$ turni sugli spalti, pronti a dare l'allarme al primo avvistamento sospetto.

Ad interrompere l'immobilità di quei giorni fu la visita inaspettata del tutore di suo figlio. Doveva per forza dirle qualcosa della massima importanza se si presentava al castello dopo che, per precauzione, lei stessa l'aveva fatto trasferire in una residenza segreta, lontano dagli artigli del Conte Palatino e da altre insidie.

"Vedervi qui m'inquieta. Se avete sfidato le intemperie e gli agguati delle strade mettendo a repentaglio la vostra sicurezza e la mia, vuol dire che siete impazzito. Oppure, che scappate da un pericolo superiore a quello appena corso per raggiungermi..." 
«Purtroppo o per fortuna, non sono mai stato più lucido di quanto non lo sia adesso, mia signora! Se ho contravvenuto alle vostre disposizioni lasciando il mio rifugio è solo perché dovevo avvertirvi subito che qui a Csejthe non siete più al sicuro..."

“Andate al punto".

"Thurzó ha forse rinunciato a combattervi con le armi, ma non illudetevi, è solo un trucco per distrarvi, mentre vi attacca alle spalle in modo subdolo, seminando veleni sul vostro conto..."

"A quale diavoleria vi riferite?"

"Ho informazioni certe sul fatto che molto presto vi trascinerà davanti ai giudici con accuse infamanti”.

"Dimenticate che non si processa facilmente una del mio rango, soprattutto se difende la libertà del suo Paese..."

"Lo credete così ingenuo da incorrere in un simile errore? In verità, ha aggirato l'ostacolo fabbricando una montagna di prove per incriminarvi su delitti che nulla hanno a che vedere con il patriottismo e la politica".

“Quali delitti?” domandò allora la Contessa, tesa come la corda di un arco.

“L'argomento è imbarazzante e Dio solo sa quanto io peni a parlarvene, ciononostante non posso fare altrimenti..."

"E allora che aspettate? Sputate il rospo senza troppi giri di parole".

"Sostiene che avete provocato la morte di centinaia di fanciulle al vostro servizio. Ma il peggio è che secondo lui l'avreste fatto per potervi poi bagnare nel loro sangue, al solo scopo di conservare la vostra bellezza più a lungo". 
Erzsébet Báthory scoppiò a ridere come non le succedeva ormai da tempo. "Chi volete che creda a una simile fandonia? La sua fantasia galoppa, ma l'intelligenza non lo sorregge..."

"Sbagliate!" la corresse il tutore, visibilmente turbato. "È ignobile e codardo, ne convengo, ma ammetterete che l'abilità non gli manca...Ha l'astuzia d'una volpe e sa come sfruttare a suo vantaggio le maldicenze che corrono sul vostro conto".

"Vi ascolto".

"Ha raccolto le confidenze di quanti vi dicono ossessionata dalla paura di invecchiare, gli stessi che diffidano dei misteriosi miscugli preparati per voi dalla vostra devotissima ancella...A questo aggiungete il fatto inconfutabile che alle vostre corti la servitù si ammala spesso e che altrettanto spesso muore..."

"Non credo a ciò che sento! Lo sanno anche i sassi che la gente povera è di costituzione debole e crepa facilmente. Succede la stessa identica cosa nei castelli di Thurzó e di altri nobili..."

"Ci sono cento testimoni pronti a giurare di avervi vista raccogliere il loro sangue, con la complicità di Anna Darvulia..."

"Razza di ignoranti! A volte, per aiutare quelle sventurate, pratico loro dei salassi, così come mi è stato insegnato dai migliori medici del regno".

"Non ditelo a me, che vi sono amico, ma preparatevi a spiegarlo a chi di dovere. I magistrati potrebbero essere inflessibili e chiedervi prove difficili da trovare".

"Prove? Quali?"

"Sapete meglio di me quanto la legge si presti agli inganni: è più facile produrre testimonianze a carico dell'imputato che non a sua discolpa...Ma adesso devo andare, se non voglio che il buio mi sorprenda lungo la strada".

"Farò buon uso delle vostre informazioni, ve lo prometto". 
"Se per allontanare da voi certi orribili sospetti foste costretta a rivoltare cielo e terra, fatelo! Costi quel che costi, dovete uscire indenne da questa brutta storia che rischia d'infangare la vostra reputazione e quella dei vostri figli..."

Quando il tutore si rimise in cammino per Sárvár, Erzsi chiamò a sé Anna Darvulia per metterla a parte del complotto ordito ai suoi danni.

"Non escludo che quel cane di Thurzó punti a screditarmi al solo scopo di potersi impossessare dei miei averi. Lo sanno pure i sassi che m'invidia le terre e che vorrebbe per sé i miei castelli..."

"Darete fuoco al Paese piuttosto che cederglieli, non è vero?" insorse l'altra, sentendosi in pericolo a sua volta. "Voi siete più potente di lui e lo schiaccerete come un verme..."

"Dovesse essere l'ultima cosa che faccio" rispose solennemente la Contessa. "Per nessuna ragione al mondo permetterò che io, una Báthory, venga anche soltanto convocata dal tribunale, senza ottenere la pelle di colui che mi ci ha trascinata!"

Quel giorno finì così, fra tuoni e fulmini, imprecazioni e desideri di vendetta.

Poi arrivò il Natale e a Csejthe si pensò soltanto a fare festa.

Per l'occasione, Erzsébet scese nelle cucine, cosa che non rientrava nelle sue abitudini. Dettò ai cuochi la minuta per un banchetto degno degli antichi sibariti: brodo di gallina e zuppa di pesce, cavoli farciti con riso e carne e costola di maiale fritta nel burro. Per dolce, un rotolo di pasta ripieno di noci e uva passa.

Al momento della cena, si presentò a tavola con un abito di seta scarlatta che le era costato quanto un anno di paga del suo contabile e che aveva indossato una volta sola, in occasione di un gran ballo a Vienna.

Per brindare all'indipendenza dell'Ungheria, fece servire il tokaji, il miglior vino conservato nelle sue cantine. Poi affidò a uno dei cortigiani il compito di declamare i versi del Balassi, il suo poeta preferito. 
In un clima euforico, tra cibi speziati, bevande inebrianti e declamazione di rime, nessuno dei commensali sentì la testa d'ariete sfondare il portone, né udì il clangore della battaglia tra chi voleva espugnare la fortezza e chi avrebbe dovuto difenderla.

A un certo punto, però, un rumore sordo fece ammutolire l'allegro convivio e qualche secondo dopo la porta del salone si spalancò violentemente.

Il Conte Palatino avanzò per primo, seguito da uno stuolo di soldati armati fino ai denti.

"Avete forse dimenticato le buone maniere? Da quando entrate in casa d'altri senza bussare?" esordì lei, cercando di mascherare lo sconcerto con l'ironia.

"Non è più tempo di sfide verbali, mia cara Erzsébet, e la mia pazienza è ormai al limite. Sono venuto fin qui solo per arrestarvi”.

"Che idiozia! Avete perso il senno?" gli tenne testa la Contessa, più sprezzante del solito. "Davvero non vorrei essere nei vostri panni quando re Mattia verrà informato di questa scellerata incursione nella mia dimora..."

"Finitela di dettare legge, cugina mia! D'ora in poi non potrete più seminare zizzania in lungo e largo per il Paese, come avete sempre fatto".

"Vi pare questo il modo di rivolgervi a una del mio rango? Denuncerò i vostri modi barbari e vi farò cacciare come un cane".

"Protesterete con i giudici quando vi chiameranno a rendere conto dei vostri crimini. Ma non vi nascondo che aspetto con ansia quel momento, quando vi sentirò balbettare la vostra versione dei fatti e poi soccombere sotto il responso della giustizia".

"Vaneggiate? Di cosa diamine state parlando?"

"Inutile continuare la commedia! Anche le pietre sanno che le vostre mani grondano del sangue di centinaia di vittime innocenti, che avete torturato e ucciso nel corso degli anni, senza un accenno di pietà né un'ombra di rimorso". 
"Quanta immaginazione per i vostri intrighi! Tentate di spaventarmi ma io non tremo: chi volete che creda alle vostre miserabili accuse?"

"Ci crederanno, ve lo assicuro. Forte del vostro nome, vi sentivate inattaccabile e avete osato l'indicibile, convinta che il pianto delle vittime non avrebbe oltrepassato le mura dei vostri castelli e dunque, non sareste mai stata chiamata a renderne conto ad alcuno. E invece, per vostra disdetta, quel pianto è giunto fino alle mie orecchie".

"Orecchie impure, pronte a ricevere le peggiori infamie da persone corrotte..."

“Tutto da dimostrare. Intanto è mia facoltà, oltre che mio dovere, consegnarvi alla giustizia terrena, affinché possiate subire la punizione che meritate. Starà poi al Padreterno destinarvi anche alle pene dell'inferno!"

Non volle ascoltarne la replica e l'afferrò per un braccio trascinandola via, senza guardarla negli occhi, mentre i suoi soldati si occupavano del resto della compagnia.

"La verità è che le mie ricchezze vi hanno sempre fatto gola e adesso vi illudete di poterci mettere le mani sopra" gridò la nobildonna, mentre l'altro la costringeva a scendere precipitosamente le scale. "Sarà una sgradevole sorpresa per voi scoprire che alla fine di tutto resterete con un pugno di mosche in mano!"

"Perché non risparmiate il fiato?"

"Malauguratamente per voi, avevo previsto le vostre mosse e mi sono affrettata a fare testamento. Sì, caro Thurzó, avete capito bene! Non c'è più niente che m'appartenga, a parte la veste che indosso. A questo punto, non vi resta che chiedervi se è valsa la pena d'imbastire un simile complotto per poi restare a bocca asciutta!"

La rivelazione fu un colpo basso per il Conte, che per la rabbia si mise ad insultare la sua prigioniera.

"Aveva ragione chi disse per primo che la strada di Satana scorre sulla lingua di Eva. . Avreste dovuto impugnare l'ago e imparare a ricamare anziché trastullarvi con le spade e immischiarvi negli affari di Stato come un uomo!" le disse a bassa voce, 
trattenendosi a malapena dal metterle le mani addosso. "Credevate davvero di poter umiliare impunemente uno con il mio prestigio? Davvero speravate che vi lasciassi libera di continuare a gettare fango sulla mia persona?"

"Il fango ce l'avevate già addosso, come una seconda pelle, e i vostri goffi tentativi di camuffarlo coi damaschi e le essenze che rubate agli ottomani, sono ridicoli oltre che vani..." gli rispose Erzsi, per niente intimorita da quanto le stava per succedere. "Ogni giorno, per ambizione e per denaro, calpestate i diritti della terra dove siete nato. Malgrado questo, continuate a considerarvi un uomo d'onore, mentre non siete che un vile pronto a vendere la patria allo straniero".

"Che triste spettacolo vedere una nobildonna ridotta in questo stato! Ambite a misurarvi con la politica ma apparire una povera illusa, superba quanto patetica".

"Siete in mala fede se negate, come infatti state facendo, che ho sempre portato avanti la mia causa osteggiando la vostra. Voi, invece, principe dei traditori, avete aspettato la morte di mio marito prima di tendermi la trappola!"

"Una donna deve saper stare al suo posto. Voi, al contrario, avete avuto l'impudenza d'infrangere quelle regole che da sempre ci governano, e di questo dovrete pagare il pegno".

L'aveva in pugno, eppure, non sembrava il vincitore, dato che non riusciva a sopportare con distacco il disprezzo che lei gli dimostrava tanto apertamente. Incapace di condurre il duello senza che il sangue gli andasse alla testa, affidò la Contessa ai soldati, raccomandandosi di non perderla mai di vista.

"Sapete già dove portarla" disse perentorio. Un attimo dopo sparì nel buio d'altre sale.

All'indomani, Erzsébet Báthory fu murata in una stanza del suo castello di Csejthe. Niente finestre, niente pertugi da cui filtrasse un filo di sole o di luce.

Le sembrò d'impazzire, provò a gridare. Poi scelse il silenzio, sperando che qualcuno, prima o poi, sposasse la sua causa e andasse a liberarla. Ma là dentro non 
sapeva più se fosse giorno oppure notte, inverno o invece estate. L'unica anima viva con cui aveva a che fare era lo sbirro che le passava i pasti attraverso un'apertura alta appena una spanna e larga mezza.

"Avvertitemi quando saremo a Natale" gli ripeteva di tanto in tanto, quasi che il saperlo potesse cambiare lo stato delle cose.

Per più di tre anni aspettò invano il processo a suo carico, convinta di potersi discolpare delle infamie e puntare finalmente l'indice contro quell'infame di Thurzó. Ma ormai, fuori da quelle mura, nessuno si occupava più di lei e il suo nome aveva finito per legarsi a filo doppio con quei crimini in realtà mai commessi e dei quali nessun giudice gliene avrebbe mai chiesto conto.

"Mia bella signora, vuoi sapere come ti chiamano adesso quelli del borgo che un tempo tu stessa strappavi alla fame in tempi di siccità e di carestia?" le chiese una mattina d'agosto la sentinella. A dispetto delle accuse che inchiodavano la nobildonna in quella cella senz'aria, lui l'ammirava, se non altro per la dignità con cui faceva fronte alle condizioni disumane in cui era tenuta.

Nella stanza regnava la penombra e attraverso la fessura che lo metteva in comunicazione con la reclusa, la guardia poteva appena scorgerla.

"Prima eri la regina del Balaton. Ora sei per tutti la iena di Csejthe, la Contessa sanguinaria, la belva dei Carpazi..." le rivelò l'uomo, che a forza di guardarla deperire tra la solitudine e le muffe s'era mosso a compassione. "La gente, si sa, ha la memoria corta e la riconoscenza ha vita breve. Però, se sei innocente, come io credo, sono sicuro che almeno Dio ti salverà".

Fu a quel punto che Erzsi, sentendo il filo di calore che trapelava dalla voce del suo carceriere, lo mise a parte d'una confidenza: "Stanotte ho fatto un sogno, terribile e sincero. E da quel sogno ho capito che non vedrò più ingiallire le foglie in autunno, né cadere la neve in inverno o fiorire i ciliegi al sole di maggio" disse senza tradire emozioni. "Dovrei rabbrividire, eppure resto calma, come l'acqua degli stagni”. 
"Mi deludi, pallida signora del castello: non è da un'indomita guerriera della tua tempra cedere alla rassegnazione come un'umile donna di campagna! Perché disperare che qualche onesto cavaliere possa prendere a cuore la tua sorte e venga a liberarti, magari approfittando del buio della notte?"

“Chimere. Confesso che anch'io ci ho creduto, forse troppo a lungo...Ma adesso è tempo di guardare in faccia la realtà, pur orribile che sia. Meglio perdere ogni speranza che coltivare inutili illusioni. Da tempo ormai il dolore mi ha raggelato l'anima e adesso è con uno strano sollievo che sento arrivare la mia ora".

"Se cedi, l'avrai data vinta a chi ti vuole morta e non potrai mai più riprenderti l'onore..."

"La verità, prima o poi, verrà a galla. Lo sa bene chi mi ha rinchiusa qui dentro. Dubito che riesca a dormire sonni tranquilli!. . . Ma in fondo, cosa vuoi che conti il passato adesso che l'avvenire sfugge?"

Esile come un giunco, con la voce tremante, Erzsébet s'accostò al muro, accanto alla piccola apertura che la teneva in contatto con il suo carceriere, e ritrovando il suo antico orgoglio gli chiese un favore: “Appena sapranno che me ne sono andata all'altro mondo, in tanti correranno a reclamare le mie spoglie. Allora, per la prima volta da che m’hanno murata in questa stanza, anche mio figlio Pál sarà autorizzato a farmi visita, sebbene ormai sarò soltanto un algido cadavere".

"Devo forse consegnargli qualcosa?"

"Mi hanno spogliata di ogni cosa, cosa potrei mai dargli se non la sudicia veste che mi ricopre?. . . Ciò che ti chiedo è invece di parlargli in privato, per riferirgli quanto io tenga che lui rispetti per filo e per segno le mie ultime volontà su questa terra".

“Ti ascolto".

Erzsi sospirò di stanchezza, forse anche di rimpianto. "Voglio più di ogni altra cosa al mondo che faccia suo un comandamento che è stato mio: l'indipendenza degli uomini e delle nazioni, un bene irrinunciabile e supremo". 
"Che altro?"

Lei sorrise, poi confessò che voleva essere seppellita sotto un maestoso pioppo bianco. E al suo interlocutore, stupito da una simile richiesta, disse sibillina: "Ha fama d'essere l'albero della morte luminosa..." Poi, quasi tra sé, a bassa voce: "Strano desiderio per una che alla luce ha sempre preferito l'ombra..."

Quando quel mattino di agosto del milleseicentoquattordici le campane del borgo suonarono l'ora sesta, lei era già morta da un pezzo. Non fu suo figlio Pál a occuparsi dei suoi miseri resti né ebbe voce in capitolo quando si trattò di decidere dove interrarli.

Di ciò che era stata Erzébet Báthory si parlò ancora a lungo, però sottovoce, alimentando le più torbide leggende sul suo conto. Nessuno, neanche in seguito, cercò mai di ristabilire la verità sulla sua storia, lasciando così che nel corso dei secoli il ricordo di una donna fuori del comune, in lotta per la libertà del suo Paese, fosse spodestato dalla fama di una creatura diabolica, assetata di sangue e di rovina. 